\title{
Reduced search space multiple shift maximum element sequential matrix diagonalisation algorithm
}

\author{
J Corr*, K Thompson*, S Weiss*, I K Proudler ${ }^{\dagger}$, J G McWhirter ${ }^{\ddagger}$ \\ ${ }^{*}$ Department of Electronic and Electrical Engineering, University of Strathclyde, Glasgow, Scotland \\ ${ }^{\dagger}$ School of Electronic, Electrical and Systems Engineering, Loughborough University, Loughborough, UK \\ ${ }^{\ddagger}$ School of Engineering, Cardiff University, Cardiff, Wales, UK \\ \{jamie.corr,keith.thompson,stephan.weiss\}@strath.ac.uk
}

Keywords: Multiple Shift Maximum Element; Sequential Matrix Diagonalisation; Polynomial Matrix Eigenvalue Decomposition.

\begin{abstract}
The Multiple Shift Maximum Element Sequential Matrix Diagonalisation (MSME-SMD) algorithm is a powerful but costly method for performing approximate polynomial eigenvalue decomposition (PEVD) for space-time covariance-type matrices encountered in e.g. broadband array processing. This paper discusses a newly developed search method that restricts the order growth within the MSME-SMD algorithm. In addition to enhanced control of the polynomial degree of the paraunitary and parahermitian factors in this decomposition, the new search method is also computationally less demanding as fewer elements are searched compared to the original while the excellent diagonalisation of MSME-SMD is maintained.
\end{abstract}

\section{Introduction}

To accurately model the delay and multipath properties of broadband array processing systems explicit lag elements must be used rather than the phase shifts employed in narrowband systems. Using delays rather than phase shifts generates a space-time covariance matrix, $\mathbf{R}[\tau]$, which captures both spatial and temporal aspects of the signals. Taking the $z$-transform of the space-time covariance matrix, $\boldsymbol{R}(z) \bullet \mathbf{R}[\tau]$, generates the polynomial cross-spectral density (CSD) matrix. The structure of the CSD matrix produced can be seen as a matrix with polynomial elements or as a polynomial that has matrices as its coefficients. The CSD matrix, $\boldsymbol{R}(z)$, is parahermitian, i.e. $\boldsymbol{R}(z)=\tilde{\boldsymbol{R}}(z)$, where the parahermitian operation, $\{\tilde{r} \cdot$, consists of a complex conjugate transpose and time reversal, such that $\tilde{\boldsymbol{R}}(z)=\boldsymbol{R}^{\mathrm{H}}\left(z^{-1}\right)$. The traditional eigenvalue decomposition (EVD) which is used in many narrowband signal processing problems is not directly applicable to the polynomial CSD matrix. The polynomial EVD (PEVD) [1] can be seen as an extension of the EVD to the polynomial matrix case. The PEVD can be used to factorise the parahermitian, $\boldsymbol{R}(z)$, into

$$
\boldsymbol{R}(z) \approx \tilde{\boldsymbol{Q}}(z) \boldsymbol{D}(z) \boldsymbol{Q}(z)
$$

where $\boldsymbol{Q}(z)$ is paraunitary such that $\boldsymbol{Q}(z) \tilde{\boldsymbol{Q}}(z)=\tilde{\boldsymbol{Q}}(z) \boldsymbol{Q}(z)=$ I and $\boldsymbol{D}(z)$ is a diagonal polynomial matrix. Although an exact decomposition in (1) cannot be guaranteed [1], [2] suggests the approximation can be accurate for sufficiently high orders of $\boldsymbol{Q}(z)$. Recently a wide variety of applications for the PEVD have arisen in areas such as broadband angle of arrival estimation [3], filter bank-based channel coding [4], subband coding [5], and the design of broadband precoding and equalisation of MIMO systems [6]. The polynomial subspace decomposition techniques, including $[4,6,3]$, require an accurate PEVD with low order paraunitary matrices to reduce the computational cost of the application. The decomposition in (1) can be calculated through a variety of different PEVD algorithms [1,7-10]. Since the PEVD is only unique to within multiplication by all-pass filters each algorithm may produce a different decomposition and each has its own merits. This paper considers the recently developed sequential matrix diagonalisation (SMD) family of algorithms [8,9]. Compared to other PEVD algorithms, such as the SBR2 algorithm, [1] the SMD methods produce decompositions exhibiting a greater degree of diagonalisation (thus greater accuracy) but are more computationally costly to implement. The original SMD algorithm [8] has been shown to give good results and the lowest order paraunitary matrices. The results of the multiple shift maximum element (MSME) SMD [9] are better in terms of convergence however the multiple shifts cause the order of the paraunitary matrices to grow faster. The aim of this paper is to restrict the order growth seen in MSME-SMD whilst maintaining a similar level of diagonalisation performance. A further benefit is that computational cost is also reduced due to less data being processed. Sec. 2. reviews the current stateof-the-art sequential matrix diagonalisation algorithms. Sec. 3. analyses the worst case polynomial order growth for the SMD and MSME-SMD algorithms. Sec. 4. then highlights the benefits of the restricted search in the MSME-SMD algorithm. Simulation results are presented in Sec. 5. to compare the different PEVD methods and conclusions are given in Sec. 6.

\section{PEVD Algorithms based on Sequential Matrix Diago- nalisation}

This section first gives an overview of the main steps involved in the SMD family of PEVD algorithms before exploring the 
SMD and MSME-SMD algorithms in detail.

The SMD family of algorithms has an initialisation step where all off-diagonal energy of the zero lag is transferred onto the diagonal via an EVD,

$$
\mathbf{S}^{(0)}[0]=\mathbf{Q}^{(0)} \mathbf{R}[0] \mathbf{Q}^{(0) \mathrm{H}} .
$$

The modal matrix $\mathbf{Q}^{(0)}(z)$ is then applied to all lags in the parahermitian matrix,

$$
\mathbf{S}^{(0)}(z)=\mathbf{Q}^{(0)} \boldsymbol{R}(z) \mathbf{Q}^{(0) \mathrm{H}} .
$$

At each iteration a generic PEVD algorithm consists of three main steps, first a search is carried out to determine which row(s) and column(s) are to be brought onto the zero lag. This search step is algorithm dependent and will be discussed in more detail below. Next the selected row(s) and column(s) are shifted onto the zero lag by means of a paraunitary shift operation,

$$
\boldsymbol{S}^{(i) \prime}(z)=\boldsymbol{\Lambda}^{(i)}(z) \boldsymbol{S}^{(i-1)}(z) \tilde{\boldsymbol{\Lambda}}^{(i)}(z), \quad i=1 \ldots I .
$$

Each PEVD iteration is then completed by bringing the offdiagonal energy at lag zero onto the diagonal, which is done by calculating and applying the modal matrix for the EVD of the zero lag to all lags of the parahermitian matrix,

$$
\boldsymbol{S}^{(i)}(z)=\mathbf{Q}^{(i)} \boldsymbol{S}^{(i) \prime}(z) \mathbf{Q}^{(i) \mathrm{H}} .
$$

All SMD algorithms stop when either a set number of iterations, $I$, have been carried out or the search step returns a value which is below a predefined threshold. Upon completion the approximately diagonal parahermitian matrix is $\boldsymbol{S}^{(I)}(z)$, and the product of the individual delay and EVD operations is used to construct the paraunitary matrix for the decomposition i.e.

$$
\boldsymbol{Q}^{(I)}(z)=\boldsymbol{G}^{(I)}(z) \ldots \boldsymbol{G}^{(1)}(z) \boldsymbol{G}^{(0)}(z),
$$

where the unitary matrices, $\boldsymbol{G}^{(i)}(z)$, are constructed from the delay and EVD operations from each of the $I$ steps,

$$
\boldsymbol{G}^{(i)}(z)=\mathbf{Q}^{(i)} \boldsymbol{\Lambda}^{(i)}(z)
$$

\subsection{Sequential Matrix Diagonalisation}

To determine which elements are brought onto the zero lag the original SMD algorithm uses a search based on column norms within the parahermitian matrix

$$
\left\{k^{(i)}, \tau^{(i)}\right\}=\arg \max _{k, \tau}\left\|\hat{\mathbf{s}}_{k}^{(i-1)}[\tau]\right\|_{2}, \quad i=1 \ldots I,
$$

where $\hat{\mathbf{s}}_{k}^{(i-1)}[\tau]$ is a modified column vector which contains all elements excluding the on-diagonal entry. Once the column with the largest norm is found it is brought onto the zero lag using the delay matrix

$$
\boldsymbol{\Lambda}^{(i)}(z)=\operatorname{diag}\{\underbrace{1 \ldots 1}_{k^{(i)}-1} z^{-\tau^{(i)}} \underbrace{1 \ldots 1}_{M-k^{(i)}}\}
$$

where the parameters $k^{(i)}$ and $\tau^{(i)}$ are the column and lag indices obtained in (8). (a)

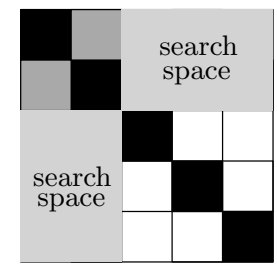

(c)

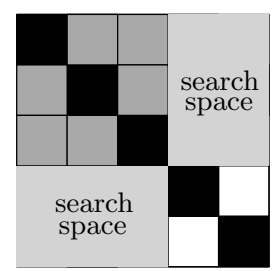

(b)

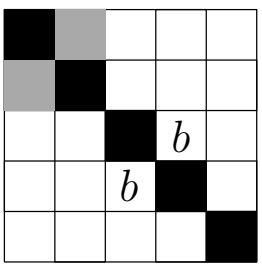

(d)

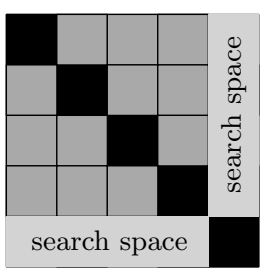

Figure 1. View of a $5 \times 5$ parahermitian matrix during the $i$ th iteration, not showing the lag dimension: starting from the top $2 \times 2$ matrix containing the maximum off-diagonal element in (a), (b) shows an example of an element resistant to permutations, the third and fourth stages of the set of reduced search space strategy are shown in (b) and (d).

\subsection{Multiple Shift Maximum Element SMD}

For the multiple shift maximum element search, step (8) is modified to use the $l_{\infty}$ rather than the $l_{2}$ norm,

$$
\left\{k^{(i)}, \tau_{k^{(i)}}^{(i)}\right\}=\arg \max _{k, \tau}\left\|\hat{\mathbf{s}}_{k}^{(i-1)}[\tau]\right\|_{\infty}, \quad i=1 \ldots I,
$$

where the symbols have the same meanings as (8). The maximum element search is carried out a total of $M-1$ times during each iteration in an attempt to maximise the amount of energy brought onto the zero lag

The parahermitian symmetry limits the maximum number of elements that can be moved onto the zero lag without affecting previous choices to $M-1$. Even then some choices result in fewer elements being able to be moved. To ensure that the full quota of $M-1$ maximum elements are transferred onto the zero lag and that the shifts do not adversely affect one another, a set of reduced search spaces is required [9]. The masks used to reduce the search spaces are shown in Fig. 1 for the case where $M=5$.

Prior to Fig. 1 (a) the first, global, maximum element is found (without any restrictions) and permuted into the top left $2 \times 2$ sub-matrix. Any element found in the search space identified in Fig. 1 (a) can be brought onto the zero lag and permuted into the top left $3 \times 3$ sub-matrix with out affecting the initial global maximum. If the second element was found in the position of element $b$ in Fig. 1 (b) it would not be possible to permute it into the upper left $3 \times 3$ sub-matrix. As a result we would have to proceed to Fig. 1 (d) meaning a total of only 3 elements could be found. Using the MSME-SMD search method, following the search space in Fig. 1 (a) and a permutation to bring a second element (from the search space in Fig. 1 (a)) into the upper left $3 \times 3$ sub-matrix, Fig.1 (c) is obtained. The search, shift and permute process is repeated using the mask in Fig. 1 (c), however this time element $b$ from Fig. 1 (b) could be chosen. Finally the fourth or $(M-1)$-th element is found using the mask in Fig. 1 (d).

In practice the permutations mentioned above are not strictly 
required, they are only used here to help illustrate the search and mask process, without permutations the search spaces simply become split up and are in different locations from Fig. 1. After the $(M-1)$ maximum elements have been located they are then transferred onto the zero lag using the delay matrix

$$
\Lambda^{(i)}(z)=\operatorname{diag}\left\{z^{-\tau_{1}^{(i)}} z^{-\tau_{2}^{(i)}} \ldots z^{-\tau_{M}^{(i)}}\right\} .
$$

\section{Polynomial Order Growth}

This section analyses the worst case polynomial order growth for both the SMD and MSME-SMD algorithms. It is likely that the actual polynomial matrix growth will be less than the worst case at each iteration. The analysis below assumes we have a parahermitian matrix, $\boldsymbol{S}^{(i)}(z)$, at the $i$-th iteration with a size of $M \times M \times 2 L+1$, i.e. the maximum lag in either direction is $|L|$.

The growth in order of the parahermitian, $\boldsymbol{S}^{(i)}(z)$, and paraunitary, $\boldsymbol{Q}^{(i)}(z)$, matrices is determined by the order of the shift matrix, $\boldsymbol{\Lambda}^{(i)}(z)$. To help analyse the problem we define the largest possible shift as $\Delta_{\max }$ and the highest possible order for the shift matrix $\Lambda_{\max }$.

In the case of the SMD algorithm the growth is simply determined by the magnitude of $\tau^{(i)}$ found in (8) which can have a maximum value of $L$, therefore $\Delta_{\max }=L$. With $\Delta_{\max }=L$ the maximum SMD shift matrix length, $\Lambda_{\max }=L$. When $\boldsymbol{\Lambda}^{(i)}(z)$ of order $\Lambda_{\max }$ is applied to $\boldsymbol{S}^{(i)}(z)$ and $\boldsymbol{Q}^{(i)}(z)$ their order will increase by $2 \Lambda_{\max }$ or in this case $2 L$. The parameter $\Lambda_{\max }$ is doubled because it is used to advance/delay a column and delay/advance a row onto the zero lag which grows the polynomial order in both directions.

For the MSME-SMD algorithm each of the $(M-1)$ shifts can potentially interact such that the maximum shift length, $\Delta_{\max }$ is $\lceil((M-1) L) / 2\rceil$. The multiple shift algorithm can both delay and advance elements onto the zerolag in a single iteration using $\Lambda^{(i)}(z)$ therefore the maximum shift matrix order, $\Lambda_{\max }$, is $2\lceil((M-1) L) / 2\rceil$. As with SMD, when $\boldsymbol{\Lambda}^{(i)}(z)$ of order $\Lambda_{\max }$ is applied to the polynomial matrices their order will increase by $2 \Lambda_{\max }$. For the MSME-SMD algorithm the worst case polynomial order growth is $4\lceil((M-1) L) / 2\rceil$. Even with reasonably small values of $M$ the multiple shift algorithm can result in a significant increase in the worst case polynomial order growth.

The growth in polynomial order can be curtailed using appropriate parahermitian [11] and paraunitary [12,13] trim functions. Both methods are permitted to remove up to a predefined threshold of energy, $\mu$, from the outer lags of the polynomial matrices. For a parahermitian matrix the trim is done symmetrically taking advantage of its parahermitian nature. The parahermitian property is also preserved by the trim function. In the case of paraunitary matrices the trim function is applied to both sides of $\boldsymbol{Q}(z)$ asymmetrically because the outer lags of a paraunitary matrix will have different energies. The paraunitary property is replaced by near-paraunitarity after the trim function is applied but the extent of this can be minimised by using the row-shift corrected trim from [13].
Table 1. Summary of worst case polynomial order growth for the different SMD variants .

\begin{tabular}{|l|c|c|c|}
\hline & SMD & MSME & RS-MSME \\
\hline \hline$\Delta_{\max }$ & $L$ & $\lceil((M-1) L) / 2\rceil$ & $L$ \\
\hline$\Lambda_{\max }$ & $L$ & $2\lceil((M-1) L) / 2\rceil$ & $2 L$ \\
\hline Ord. Growth & $2 L$ & $4\lceil((M-1) L) / 2\rceil$ & $4 \mathrm{~L}$ \\
\hline
\end{tabular}

To reduce computational costs of the PEVD algorithms the parahermitian truncation can be carried out at the end of every iteration, the resulting maximum total loss in energy after $I$ iterations is $I \times \mu_{P H}$. As the paraunitary matrix is only ever calculated when the PEVD is complete the trim function is only applied once and so the resulting energy loss has a maximum of $\mu_{P U}$.

\section{Restricted Search MSME-SMD}

In the restricted search (RS) MSME-SMD we impose an extra condition on the search spaces in Fig. 1 to control the polynomial order growth in $\boldsymbol{S}^{(i)}(z)$ and $\boldsymbol{Q}^{(i)}(z)$. Rather than allowing every search to select elements from any lag, we restrict it to elements closer to the zero lag than the global maximum, found during the first search of each iteration. The new approach still uses (10) but now once the first search of the $i$-th iteration finds a maximum element on $\tau_{k^{(i)}}^{(i)}$, the lag parameter, $\tau$, in (10) is restricted such that $|\tau| \leq\left|\tau_{k^{(i)}}^{(i)}\right|$ for the remaining searches in the $i$-th iteration. Using this method the worst case maximum shift, $\Delta_{\max }$, is $L$, the maximum order for the shift matrix, $\Lambda_{\max }$, is $2 L$ and the polynomial order growth is $4 L$. For comparison the maximum shift, shift matrix order and polynomial order growth are summarised in Tab. 1 for all three SMD variants. The worst case scenario sees the RS-MSME-SMD order grow twice as fast as SMD but this is significantly lower than the original MSME-SMD, especially when the matrix width $M$ is increased.

Ultimately limiting the search space to lower lags will result in missing some elements and slow the algorithm's convergence slightly but these missed elements are likely to be found by searches during future iterations. The reduced search space will benefit the real time performance in two ways; first the searches during one iteration where the restriction, $|\tau| \leq\left|\tau_{k^{(i)}}^{(i)}\right|$, is applied will be on fewer elements and second the slower growth in parahermitian matrix means searches and matrix operations in future iterations will be over fewer elements.

\section{Results}

To illustrate the performance of the different PEVD algorithms we first present the performance metrics, followed by the simulation set up. Finally the results are presented and the performance of the PEVD algorithms is analysed.

\subsection{Performance Metrics}

To confirm that the RS-MSME-SMD shifts a similar amount of energy at each iteration as the original MSME-SMD, the first 
test will measure diagonalisation performance: the remaining off-diagonal energy after $i$ iterations normalised by the energy in the initial parahermitian matrix, $\mathbf{R}[\tau]$,

$$
E_{\text {norm }}^{(i)}=\frac{\sum_{\tau} \sum_{k=1}^{M}\left\|\hat{\mathbf{s}}_{k}^{(i)}[\tau]\right\|_{2}^{2}}{\sum_{\tau}\|\mathbf{R}[\tau]\|_{\mathrm{F}}^{2}},
$$

where $\|\cdot\|_{\mathrm{F}}$ represents the Frobenius norm and the vector, $\hat{\mathbf{s}}_{k}^{(i)}[\tau]$, in the numerator is the same as that used in (8) which contains all but the on diagonal elements.

The main objective of the search space restriction discussed in this paper is to limit the order growth in the polynomial matrix. With this in mind the order of the parahermitian and paraunitary matrices are recorded after each iteration.

An added benefit of the reduction in parahermitian matrix order is a reduction in the computational cost of calculating the PEVD. Here we use execution time as a measure of the computational complexity of the PEVD algorithms implemented in Matlab 2014a with the following system specification: Ubuntu 14.04 on a workstation with Intel $\AA$ Xeon $\AA$ E5-1607V2 3.00 $\mathrm{GHz} \times 4$ cores and 8 GB RAM.

\subsection{Simulation Set Up}

The results were obtained using an ensemble of $10^{3}$ parahermitian matrices produced using the source model from [14] where the source model is not majorised and has an average dynamic range of approximately $30 \mathrm{~dB}$. The source model is randomised so that the parahermitian matrices produced are unique for each instantiation. The parahermitian matrix, $\boldsymbol{R}(z)$, is $\boldsymbol{R}(z) \in \mathbb{C}^{6 \times 6}$ with the initial number of lags set to 119 . Each of the PEVD algorithms was run for 200 iterations with the performance metrics recorded after each iteration. The simulations are first run using $\mu_{P H}=\mu_{P U}=0$, i.e. only removing zero filled lags, then repeated over the same ensemble for $\mu_{P H}=\mu_{P U}=10^{-6}$.

\subsection{Algorithm Convergence}

Fig. 2 shows the reduction in off-diagonal energy vs. algorithm iterations for the SMD algorithm and the two versions of MSME-SMD. Despite the reduced search space we can see for the example in Fig. 2 both MSME-SMD algorithms transfer a similar amount of energy at each iteration and follow an almost identical convergence curve. The amount of energy transferred by both MSME-SMD algorithms is also significantly higher than the SMD method.

As discussed in Sec. 4. the original MSME-SMD transfers marginally more energy per iteration than the new RS-MSMESMD algorithm in Fig. 2 however these are still significantly better than convergence of the SMD approach.

\subsection{Paraunitary/Parahermitian Matrix Order}

This section investigates one of the main goals of the RSMSME-SMD algorithm which is to reduce the growth in polynomial order of the parahermitian and paraunitary matrices.

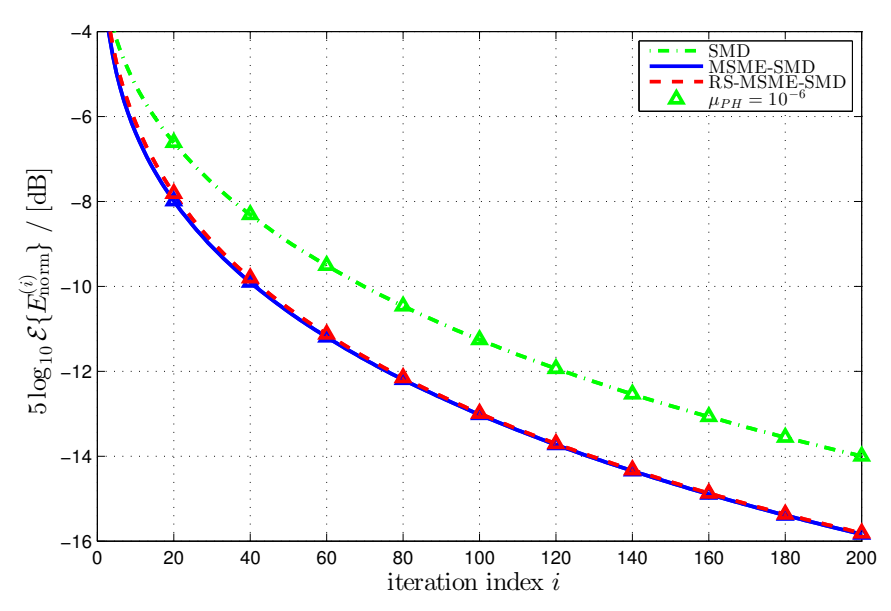

Figure 2. Diagonalisation vs. algorithm iterations for the SMD algorithm and the two MSME-SMD varients.

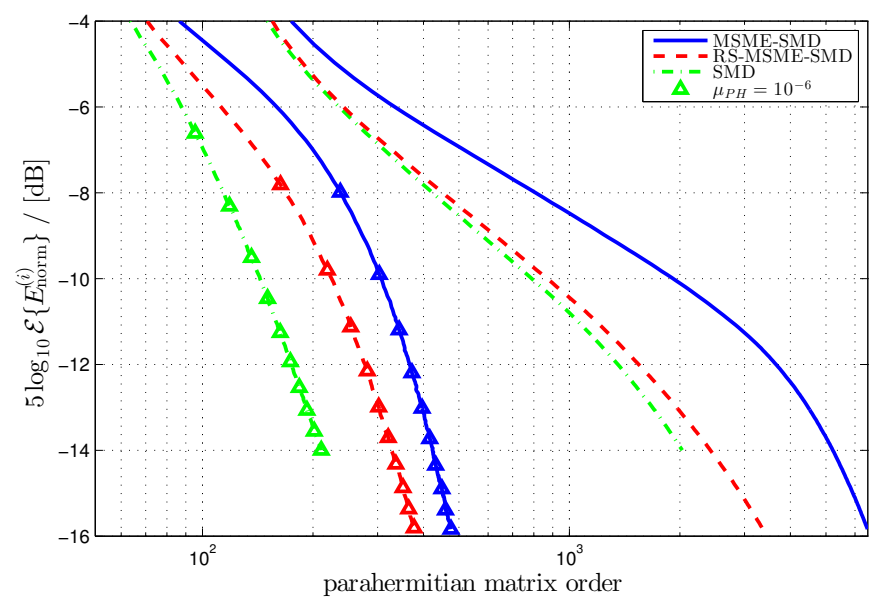

Figure 3. Reduction in off-diagonal energy vs. growth in parahermitian matrix order.

Figs. $3 \& 4$ show the order growth of the parahermitian and paraunitary matrices for each of the selected PEVD algorithms. Generally the SMD method produces parahermitian and paraunitary matrices of lower order than any of the other PEVD methods [10]. Here we can see in both Fig. $3 \& 4$ that the matrices produced by RS-MSME-SMD are significantly shorter than their MSME-SMD equivalent and are a similar level to SMD. Even when a truncation algorithm such as those described in [11] and [13] are applied to the parahermitian and paraunitary matrices the reduced search method still outperforms the original MSME-SMD as shown in Fig. $3 \& 4$ although it does lose out slightly to SMD.

\subsection{Real Time Execution}

Fig. 5 shows the time taken for each of these algorithms to carry out 200 iterations alongside the diagonalisation measure at each point. Despite their more complex search methods the MSME-SMD algorithms actually converge faster in real time than the SMD approach in all cases. When no truncation is used we can see that the new reduced search MSME method 


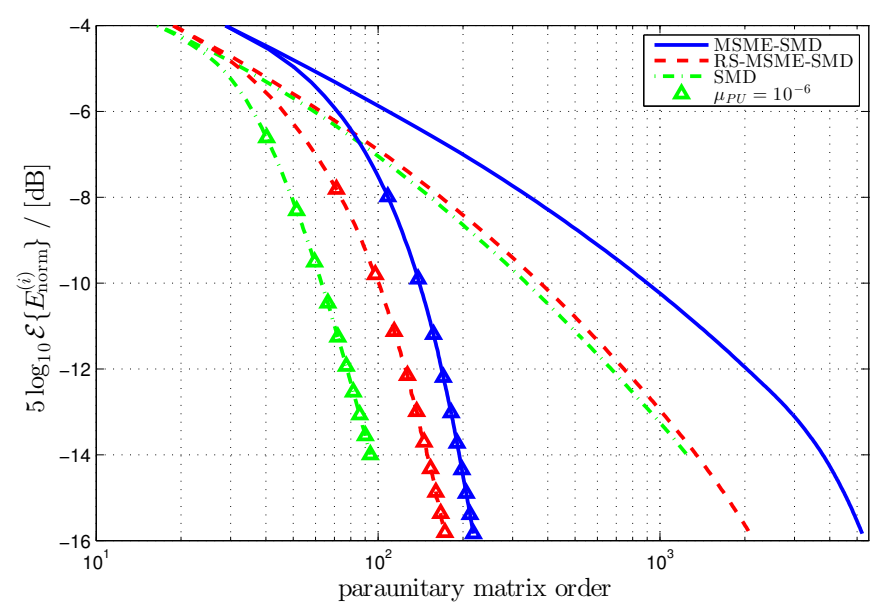

Figure 4. Reduction in off-diagonal energy vs. growth in paraunitary matrix order.

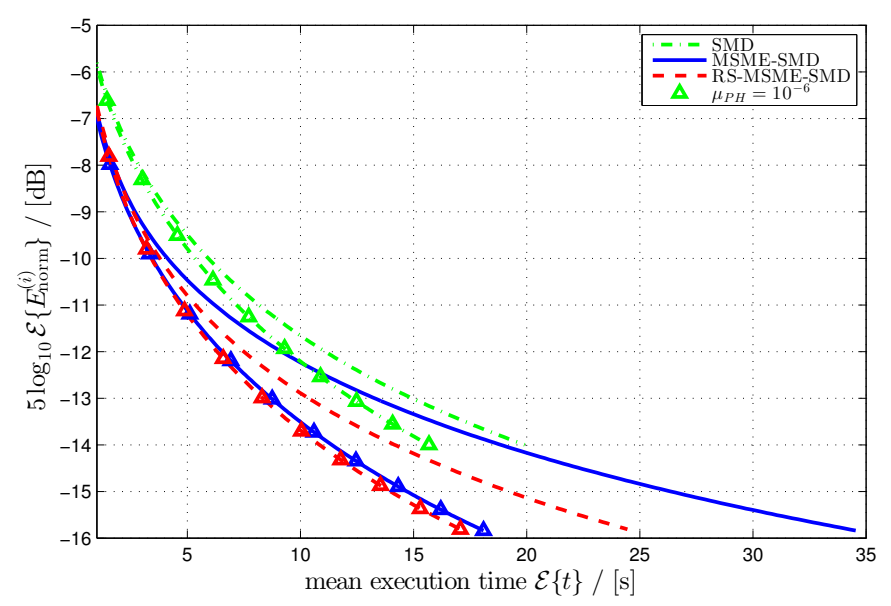

Figure 5. real time convergence of PEVD algorithms, diagonalisation measure vs. mean execution time.

is more efficient than the original MSME search, in fact the new method takes on average around 10 seconds less than its predecessor to complete 200 iterations. When the parahermitain truncation methods are included both MSME-SMD variants obtain a significant performance improvement, whereas the same change in SMD has a lesser effect. The performance benefits of the reduced search MSME-SMD are not as obvious when the parahermitian truncation is used but it still performs better than the original MSME-SMD.

\section{Conclusion}

Through analysis of the polynomial order growth of the SMD and MSME-SMD algorithms we have proposed a new search method which can significantly reduce the polynomial order growth of the MSME-SMD algorithm. Results indicate almost no degradation in energy transfer between the existing and proposed method. In addition the experiments have shown that the new method leads to a significant reduction in polynomial matrix order growth even when truncation methods are used. The reduced search spaces and resulting lower order paraher- mitian matrices also result in an improved real time convergence. When the parahermitian and paraunitary matrices are truncated the benefits of the new search method are reduced. In general the restriction of the search space slows the growth of both paraunitary and parahermitian matrices which leads to a faster execution time with minimal impact on the algorithm convergence.

\section{Acknowledgement}

This work was supported by the Engineering and Physical Sciences Research Council (EPSRC) Grant number EP/K014307/1 and the MOD University Defence Research Collaboration in Signal Processing.

\section{References}

[1] J.G. McWhirter, P.D. Baxter, T. Cooper, S. Redif, J. Foster. An EVD Algorithm for Para-Hermitian Polynomial Matrices. IEEE Trans. SP, 55(5):2158-2169, May 2007.

[2] S. Icart, P. Comon. Some Properties of Laurent Polynomial Matrices. In Conf. Math. Signal Proc., Birmingham, UK, Dec. 2012.

[3] M. Alrmah, S. Weiss, S. Lambotharan. An Extension of the MUSIC Algorithm to Broadband Scenarios using Polynomial Eigenvalue Decomposition. In EUSIPCO, pp. 629-633, Barcelona, Spain, Aug. 2011.

[4] S. Weiss, S. Redif, T. Cooper, C. Liu, P.D. Baxter, J.G. McWhirter. Paraunitary Oversampled Filter Bank Design for Channel Coding. J. Applied SP, 2006.

[5] S. Redif, J. McWhirter, S. Weiss. Design of FIR Paraunitary Filter Banks for Subband Coding using a Polynomial Eigenvalue Decomposition. IEEE Trans. SP, 59(11):5253-5264, Nov. 2011.

[6] C.H. Ta, S. Weiss. A Design of Precoding and Equalisation for Broadband MIMO Systems. In 15th Int. Conf. DSP, pp. 571574, Cardiff, UK, July 2007.

[7] A. Tkacenko. Approximate Eigenvalue Decomposition of ParaHermitian Systems through Successive FIR Paraunitary Transformations. In IEEE ICASSP, pp. 4074-4077, Dallas, TX, Mar. 2010

[8] S. Redif, S. Weiss, J. McWhirter. Sequential Matrix Diagonalization Algorithms for Polynomial EVD of Parahermitian Matrices. IEEE Trans. SP, 63(1):81-89, Jan. 2015.

[9] J. Corr, K. Thompson, S. Weiss, J. McWhirter, S. Redif, I. Proudler. Multiple Shift Maximum Element Sequential Matrix Diagonalisation for Parahermitian Matrices. In IEEE SSP, pp. 312-315, Gold Coast, Australia, June 2014.

[10] J. Corr, K. Thompson, S. Weiss, I. Proudler, and J. McWhirter. Causality-Constrained Multiple Shift Sequential Matrix Diagonalisation for Parahermitian Matrices In EUSIPCO, Lisbon, Portugal, September 2014.

[11] J. Foster, J.G. McWhirter, J. Chambers. Limiting The Order of Polynomial Matrices within the SBR2 Algorithm. In IMA Math. Signal Proc., Cirencester, UK, Dec. 2006.

[12] C.H. Ta, S. Weiss. Shortening the Order of Paraunitary Matrices in SBR2 Algorithm. In Int. Conf. Inf. Comm. \& SP, pp. 1-5, Singapore, Dec. 2007.

[13] J. Corr, K. Thompson, S. Weiss, I. Proudler, and J. McWhirter. Row-Shift Corrected Truncation of Paraunitary Matrices for PEVD Algorithms. In EUSIPCO, Nice, France, September 2015.

[14] J. Corr, K. Thompson, S. Weiss, I. Proudler, and J. McWhirter. Impact of source model matrix conditioning on iterative PEVD algorithms In IET Inteligent Signal Processing Conference, London, England, December 2015. Submitted. 